M. Nakai

Nagoya Math. J.

Vol. 70 (1978), 7-24

\title{
PICARD PRINCIPLE FOR FINITE DENSITIES
}

\author{
MITSURU NAKAI
}

A nonnegative locally Hölder continuous function $P(z)$ on $0<|z| \leq 1$ will be referred to as a density on $\Omega: 0<|z|<1$ with singularity at $\delta: z=0$, removable or genuine. A density $P$ on $\Omega$ is said to be finite if it is integrable over $\Omega$ :

$$
\int_{\Omega} P(z) d x d y<\infty
$$

The elliptic dimension of a density $P$ on $\Omega$ at $\delta, \operatorname{dim} P$ in notation, is defined to be the dimension of the half module of nonnegative solutions of the equation $\Delta u=P u$ on $\Omega$ with vanishing boundary values on $|z|=1$. After Bouligand we say that Picard principle is valid for a density $P$ at $\delta$ if $\operatorname{dim} P=1$. The purpose of this paper is to establish the following

MAIN THEOREM. The Picard principle is valid for any finite density $P$ on $\Omega$ at $\delta$.

The proof of this will be given in no. 16 after a series of preparations in nos. 1-15. First we discuss the convex structure of the space of nonnegative solutions and introduce the Martin compactification of $\Omega$ relative to a density in nos. 1-5. The Martin representation theorem as well as the precise definition of elliptic dimensions will then be given in no. 6. A duality theorem between the Picard principle and the Riemann theorem is given in nos. 7-8, which is one of chief tools for the main theorem. Two propositions concerning limit properties at $\delta$ of functions in $\Omega$ with square integrable gradients discussed in nos. 9-12 may have independent interests. Two conditions in terms of the $P$-unit from which the Picard principle follows will be given in nos. 13-14. The key inequality for the proof of the main theorem will be derived in no. 15 and then the proof will be completed in no. 16. A relation between our

Received March 17, 1975. 
present condition and our former one in [11] for the validity of Picard principle is remarked in the final no. 17. For an elementary exposition on fundamentals of the equation $\Delta u=P u$ such as the Dirichlet problem and the Green's function we refer e.g. to no. 1 in [11].

We shall consider $\Omega: 0<|z|<1$ as an end (a subsurface of a Riemann surface with a compact relative boundary and a single ideal boundary component) of the punctured extended plane $\hat{C}_{0}: 0<|z| \leq \infty$. Therefore the unit circle $|z|=1$ is viewed as the relative boundary $\partial \Omega$ of $\Omega$ and the origin $z=0$ as an ideal boundary $\delta=\delta \Omega$ of $\Omega$. We also use the notation $\bar{\Omega}$ for the closure of $\Omega$ in $\hat{C}_{0}$, i.e. $\bar{\Omega}=\Omega \cup \partial \Omega: 0<|z| \leq 1$. As usual $\Delta \varphi(z)=\partial^{2} \varphi(z) / \partial x^{2}+\partial^{2} \varphi(z) / \partial y^{2}$ for $\varphi \in C^{2}$ and $\nabla \varphi(z)=(\partial \varphi(z) / \partial x, \partial \varphi(z) / \partial y)$ for $\varphi \in C^{1}$ or more generally for piecewise smooth $\varphi$ with $z=x+i y$. We remark that most parts of the discussions in the sequel are obviously valid for a general end $\Omega$ and a density $P$ (i.e. nonnegative Hölder continuous 2-form $P(z) d x d y$ ) but whether the main theorem is valid for a general end of harmonic dimension one and a finite density is unsettled. The most general result in our frame would be: The elliptic dimension of any finite density on an end at the ideal boundary coincides with the harmonic dimension of the end.

1. We denote by $\mathscr{P}$ the set of nonnegative solutions $u$ of $L_{P} u \equiv$ $\Delta u-P u=0$ on $\Omega$ with boundary values zero on $\partial \Omega$ and by $\mathscr{E}$ the space of solutions $v$ of $L_{P} u=0$ on $\Omega$ such that $|v|$ is dominated by a $u$ in $\mathscr{P}$. In other words $\mathscr{E}=\mathscr{P} \ominus \mathscr{P}=\left\{u_{1}-u_{2} ; u_{j} \in \mathscr{P}(j=1,2)\right\}$. It is easily checked that $\mathscr{E}$ is a locally convex topological vector space with respect to the topology on $\mathscr{E}$ induced by the convergence on each compact subset of $\Omega$. The set $\mathscr{P}$ is a closed cone in $\mathscr{E}$. The order on $\mathscr{E}$ induced by $\mathscr{P}$, i.e. $u \geq v$ if $u-v \in \mathscr{P}$, is the usual pointwise function ordering. We denote by $u \vee v(u \wedge v$, resp.) the least majorant (greatest minorant, resp.) of $u$ and $v$ in $\mathscr{E}$ if it exists. We first maintain: The space $\mathscr{E}$ forms a vector lattice with lattice operations $\vee$ and $\wedge$. We only have to show that for any $u \in \mathscr{E}$ there exists $u \vee 0$ in $\mathscr{E}$. Then $(u-v) \vee 0+v$ and $-((-u) \vee(-v))$ are the required $u \vee v$ and $u \wedge v$ in $\mathscr{E}$ for any $u$ and $v$ in $\mathscr{E}$. Since there exists a $w \in \mathscr{P}$ such that $u \leq|u| \leq w$, the set of majorants of $u$ and zero in $\mathscr{E}$ is nonvoid. Let $w$ be any majorant of $u$ and zero in $\mathscr{E}$. Then $w \geq u \cup 0=\max (u, 0)$. We denote by $v_{t}$ the solution of $L_{P} u=0$ on $t<|z|<1$ with boundary values zero on $|z|=1$ and $u \cup 0$ on $|z|=t(0<t<1)$. By the maximum principle $w \geq v_{t} \geq u \cup 0$ and a 
fortiori $w \geq v_{s} \geq v_{t}(0<s<t)$. By the Harnack principle $\left\{v_{t}\right\}(0<t<1)$ converges to a solution $v$ of $L_{P} u=0$ on $\Omega$ as $t \rightarrow 0$ such that $w \geq v \geq$ $u \cup 0$. This shows that $v \in \mathscr{E}$ and $v=u \vee 0$.

2. The topology in $\mathscr{E}$ is metrizable, i.e. it is induced by the Fréchet norm $\|u\|=\sum_{1}^{\infty} \varepsilon_{n}\left(\sup _{|z|=r_{n}}|u(z)|\right)^{*}$ for $u \in \mathscr{E}$, where $\alpha^{*}=\alpha /(1+\alpha)$ for real numbers $\alpha \geq 0$ and $\infty^{*}=1,\left\{\varepsilon_{n}\right\}$ a sequence of positive numbers with $\sum_{1}^{\infty} \varepsilon_{n}=1$, and $\left\{r_{n}\right\}$ a strictly decreasing zero sequence in $(0,1)$. Therefore, to see that the linear functional

$$
\sigma(u)=\int_{0}^{2 \pi}\left[\frac{\partial}{\partial r} u\left(r e^{i \theta}\right)\right]_{r=1} d \theta
$$

on $\mathscr{E}$ is continuous, we only have to show $\lim _{n \rightarrow \infty} \sigma\left(u_{n}\right)=0$ if $\left\|u_{n}\right\| \rightarrow 0$. Since $\left\|u_{n}\right\| \rightarrow 0$ implies that $\left\{u_{n}\right\}$ converges to zero uniformly on $r_{1} \leq|z|$ $\leq 1$, the sequence $\left\{\left[\partial u_{n} / \partial r\right]_{r=1}\right\}$ also converges to zero uniformly on $[0,2 \pi]$ and a fortiori $\sigma\left(u_{n}\right) \rightarrow 0$. We denote by $\mathscr{P}_{1}$ the intersection of $\mathscr{P}$ with the closed hyperplane $\{u \in \mathscr{E} ; \sigma(u)=-1\}$ in $\mathscr{E}$, i.e.

$$
\mathscr{P}_{1}=\{u \in \mathscr{P} ; \sigma(u)=-1\} \text {. }
$$

It is compact in $\mathscr{E}$ by the Harnack principle. Therefore $\mathscr{P}_{1}$ is a compact convex subset of $\mathscr{E}$. Clearly $\mathscr{P}_{1}$ is a base of the cone $\mathscr{P}$, i.e. for any $u \in \mathscr{P}$ with $u \neq 0$ there exists a unique $v \in \mathscr{P}_{1}$ and a positive number $\lambda$ such that $u=\lambda v$. Since the linear space generated by the cone with the base $\mathscr{P}_{1}$ is $\mathscr{E}$ which is a vector lattice as we saw in no. $1, \mathscr{P}_{1}$ is, by definition, a simplex. Therefore, by the Choquet representation theorem (cf. e.g. Phelps [16]) there exists a unique probability measure $\nu$ on ex $\left[\mathscr{P}_{1}\right]$, the set of extreme points of $\mathscr{P}_{1}$, for any $u \in \mathscr{P}_{1}$ such that

$$
u=\int_{\mathrm{ex}\left[\mathscr{F}_{1}\right]} v d \nu(v) .
$$

3. We denote by $\mathscr{B}$ the space of bounded solutions $u$ of $L_{P} u=0$ on $\Omega$ with continuous boundary values on $\partial \Omega$. We show that $\sup _{\bar{\Omega}}|u|=$ $\max _{\partial \Omega}|u|$. For the aim observe that $\Delta u^{2}=2\left(P u^{2}+|\nabla u|^{2}\right) \geq 0$ on $\Omega$. Therefore $u^{2}$ is bounded subharmonic on $\Omega$ and a fortiori on the whole disk $|z|<1$. By the maximum principle for subharmonic functions, we see that $u(z)^{2} \leq \max _{\partial \Omega} u^{2}$ on $|z|<1$, and the above identity is deduced. Next let $f \in C(\partial \Omega)$ with $f \geq 0$ and $u_{t}$ be the solution of $L_{P} u=0$ on $0<t<$ $|z|<1$ with boundary values $f$ on $|z|=1$ and zero on $|z|=t$. The sequence 
$\left\{u_{t}\right\}$ is increasing as $t \rightarrow 0$ and thus $u=\lim _{t \rightarrow 0} u_{t}$ is a bounded nonnegative solution of $L_{P} u=0$ on $\Omega$ with boundary values $f$ on $\partial \Omega$. By the first observation in this no. such a $u$ is unique. Therefore $u \rightarrow u \mid \partial \Omega$ is a bijective, linear, order preserving, and isometric mapping of $\mathscr{B}$ onto $C(\partial \Omega)$ with norms $\sup _{\bar{\Omega}}|u|$ in $\mathscr{B}$ and $\max _{\partial \Omega}|f|$ in $C(\partial \Omega)$. The function $e=e_{P}$ j.n $\mathscr{B}$ with $e \mid \partial \Omega=1$ is referred to as the $P$-unit.

4. The Green's function $G(z, \zeta)$ of $L_{P} u=0$ on $\Omega$ with pole $\zeta \in \Omega$ is the minimal positive solution of the Poisson equation $L_{P} u=-2 \pi \delta_{\zeta}$ (the Dirac $\delta)$. Then $G(z, \zeta)=G(\zeta, z), G(\cdot, \zeta)=0$ on $\partial \Omega, G(\cdot, \zeta)$ is a bounded solution of $L_{P} u=0$ on $\Omega$ except for an arbitrary disk about $\zeta$, and $G(z, \zeta)+\log |z-\zeta|=O(1)$ as $z \rightarrow \zeta$. Consider

$$
K(z, \zeta)=\frac{1}{2 \pi} \frac{G(z, \zeta)}{e(\zeta)}
$$

for $(z, \zeta) \in \bar{\Omega} \times \bar{\Omega}$. Applying the result in no. 3 to the region $\Omega_{t}=$ $\{\zeta ; 0<|\zeta|<t\}(0<t<|z|<1)$ we see that $0<\inf _{\zeta \in \Omega_{t}} K(z, \zeta) \leq \sup _{\zeta \in \Omega_{t}} K(z, \zeta)$ $<\infty$. Clearly $\lim _{\zeta \in \Omega, \zeta \rightarrow \partial \Omega} K(z, \zeta)=0$. Let $\rho$ be a metric on $\Omega$ given by

$$
\rho\left(\zeta_{1}, \zeta_{2}\right)=\sum_{n=1}^{\infty} \varepsilon_{n}\left(\max _{|z|=r_{n}}\left|K\left(z, \zeta_{1}\right)-K\left(z, \zeta_{2}\right)\right|\right)^{*}
$$

for $\zeta_{1}$ and $\zeta_{2}$ in $\Omega$, where $\left\{\varepsilon_{n}\right\},\left\{r_{n}\right\}$, and $*$ are as in no. 2. The topology on $\Omega$ induced by $\rho$ is homeomorphic to the usual plane topology on $\Omega$. By the above observations, $\rho$-Cauchy sequences $\left\{\zeta_{n}\right\}$ in $\Omega$ are classified into three categories: $\left\{\zeta_{n}\right\}$ converging to $\partial \Omega ;\left\{\zeta_{n}\right\}$ converging to a $\zeta \in \Omega$; $\left\{\zeta_{n}\right\}$ converging to $\zeta=0$. We consider the completion of $\Omega$ toward $\zeta=$ 0 , i.e. adding ideal elements $\zeta^{*}$ of equivalence classes of $\rho$-Cauchy sequences of the third category. We denote by $\beta=\beta_{P}$ the set of ideal elements in the completion of $\Omega$ toward $\zeta=0$. The metric $\rho$ is naturally extended to $\beta \cup \Omega$ and if $\rho\left(\zeta, \zeta^{*}\right) \rightarrow 0$ for $\zeta \in \Omega$ and $\zeta^{*} \in \beta$, then $K(z, \zeta)$ converges to a $u \in \mathscr{P}$ which we denote by $K\left(z, \zeta^{*}\right)$, i.e.

$$
K\left(z, \zeta^{*}\right)=\lim _{\zeta \in \Omega, \zeta \rightarrow \zeta^{*}} K(z, \zeta) .
$$

Then the identity (5) is also valid if $\zeta_{1}$ and $\zeta_{2}$ are in $\beta \cup \Omega$. As a consequence of the Harnack principle, $\beta$ is compact in $\beta \cup \Omega$ and the class

$$
\mathscr{K}=\left\{K\left(\cdot, \zeta^{*}\right) ; \zeta^{*} \in \beta\right\}
$$

is also compact in the locally convex space $\mathscr{E}$, and then the space $\beta \cup \Omega$ 
$\cup \partial \Omega=\beta \cup \bar{\Omega}$ is compact. We call $\Omega^{*}=\Omega_{P}^{*}=\beta \cup \Omega \cup \partial \Omega$ the Martin compactification of $\Omega$ relative to $P, \beta=\beta_{P}$ the Martin ideal boundary relative to $P$, and $K\left(z, \zeta^{*}\right)\left(\left(z, \zeta^{*}\right) \in \bar{\Omega} \times \Omega^{*}\right)$ the Martin kernel relative to $P$. Observe that

$$
e(\zeta)=\frac{-1}{2 \pi} \int_{0}^{2 \pi}\left[\frac{\partial}{\partial r} G\left(r \mathrm{e}^{i \theta}, \zeta\right)\right]_{r=1} d \theta
$$

for $\zeta \in \Omega$ (cf. nos. 3 and 8 below). This is equivalent to

$$
\int_{0}^{2 \pi}\left[\frac{\partial}{\partial r} K\left(r \mathrm{e}^{i \theta}, \zeta\right)\right]_{r=1} d \theta=-1
$$

In view of (5) the right hand side of the above is continuous on $\beta \cup \Omega$ in $\zeta$ and a fortiori we conclude that $\sigma\left(K\left(\cdot, \zeta^{*}\right)\right)=-1$ (cf. (2)), i.e.

$$
\mathscr{K} \subset \mathscr{P}_{1} .
$$

5. We denote by $\overline{c o}[\mathscr{K}]$ the closure of the convex hull of $\mathscr{K}$ in $\mathscr{E}$. Since $\mathscr{P}_{1}$ is compact and convex, the inclusion $\overline{\mathrm{co}}[\mathscr{K}] \subset \mathscr{P}_{1}$ is clear, and in fact we have

$$
\overline{\operatorname{co}}[\mathscr{K}]=\mathscr{P}_{1} .
$$

For the purpose let $\Omega_{t}$ be $0<|z|<t(0<t \leq 1), G_{t}(z, \zeta)$ the Green's function of $L_{P} u=0$ on $\Omega-\bar{\Omega}_{t}$, and $u \in \mathscr{P}_{1}$. Fix an arbitrary point $z \in \Omega$ and take $t$ so small that $z \in \Omega-\bar{\Omega}_{t}$. Applying the Green formula to $G(z, \zeta)$ and $u(\zeta)$ for the region $\Omega-\bar{\Omega}_{t}$ less an $\varepsilon$-disk about $z$ and then letting $\varepsilon \rightarrow 0$, we obtain

$$
2 \pi u(z)=\int_{0}^{2 \pi}\left[u\left(r \mathrm{e}^{i \theta}\right) \frac{\partial}{\partial r} G\left(z, r \mathrm{e}^{i \theta}\right)-G\left(z, r \mathrm{e}^{i \theta}\right) \frac{\partial}{\partial r} u\left(r \mathrm{e}^{i \theta}\right)\right]_{r=t} t d \theta .
$$

Let $v_{s}$ be the solution of $L_{P} u=0$ on $\Omega_{t}-\bar{\Omega}_{s}(0<s<t)$ with boundary values $u$ on $\partial \Omega_{t}$ and zero on $\partial \Omega_{s}$. Again by the Green formula applied to $G_{s}(z, \zeta)$ and $u(\zeta)$ for the region $\Omega_{t}-\bar{\Omega}_{s}$ we derive

$$
0=\int_{0}^{2 \pi}\left[v_{s}\left(r \mathrm{e}^{i \theta}\right) \frac{\partial}{\partial r} G_{s}\left(z, r \mathrm{e}^{i \theta}\right)-G_{s}\left(z, r \mathrm{e}^{i \theta}\right) \frac{\partial}{\partial r} v_{s}\left(r \mathrm{e}^{i \theta}\right)\right]_{r=t} t d \theta .
$$

Since $v_{s}$ converges increasingly to a solution $u_{t} \leq u$ on $\Omega_{t}$ with boundary values $u$ on $\partial \Omega_{t}$ and $G_{s}(z, \zeta)$ converges increasingly to $G(z, \zeta)$ on $\bar{\Omega}_{t}$ as $s \rightarrow 0$, we see that 


$$
0=\int_{0}^{2 \pi}\left[u\left(r \mathrm{e}^{i \theta}\right) \frac{\partial}{\partial r} G\left(z, r \mathrm{e}^{i \theta}\right)-G\left(z, r \mathrm{e}^{i \theta}\right) \frac{\partial}{\partial r} u_{t}\left(r \mathrm{e}^{i \theta}\right)\right]_{r=t} t d \theta
$$

Subtracting the above from (10) we deduce

$$
2 \pi u(z)=\int_{0}^{2 \pi}\left[G\left(z, r \mathrm{e}^{i \theta}\right) \frac{\partial}{\partial r}\left(u_{t}\left(r \mathrm{e}^{i \theta}\right)-u\left(r \mathrm{e}^{i \theta}\right)\right)\right]_{r=t} t d \theta
$$

Since $u_{t}-u=0$ on $\partial \Omega_{t}$ and $u_{t}-u \leq 0$ on $\Omega_{t}$, we see that

$$
d \mu_{t}(\zeta)=\frac{1}{2 \pi} e(\zeta)\left[\frac{\partial}{\partial r}\left(u_{t}\left(r \mathrm{e}^{i \theta}\right)-u\left(r \mathrm{e}^{i \theta}\right)\right]_{r=t} t d \theta, \quad \zeta=t e^{i \theta},\right.
$$

is a positive measure on $\Omega^{*}$ with its support in $\partial \Omega_{t}$ and (11) takes the form

$$
u(z)=\int_{\partial \Omega_{t}} K(z, \zeta) d \mu_{t}(\zeta)
$$

Applying $\sigma$ to the both sides of (12) and by using (7) and the Fubini theorem, we see that the total mass of $\mu_{t}$ is one. We can then choose a subsequence $\left\{\mu_{t_{k}}\right\}\left(t_{1}>t_{2}>\ldots>t_{k} \rightarrow 0\right)$ converging to a probability measure $\mu$ on $\Omega^{*}$ with its support in $\beta$ weakly in $C\left(\Omega^{*}\right)^{*}$. Since $K(z, \cdot)$ is continuous on $\Omega^{*}$, we deduce by letting $k \rightarrow \infty$ in (12) with $t=t_{k}$ that

$$
u(z)=\int_{\beta} K\left(z, \zeta^{*}\right) d \mu\left(\zeta^{*}\right) .
$$

The right hand side of the above belongs to $\overline{c o}[\mathscr{K}]$ and we conclude that (9) is valid.

6. The Milman theorem (cf. e.g. Dunford-Schwartz [3]) says that (9) implies ex $\left[\mathscr{P}_{1}\right] \subset \overline{\mathscr{K}}$. Since $\overline{\mathscr{K}}=\mathscr{K}$, we conclude that

$$
\operatorname{ex}\left[\mathscr{P}_{1}\right] \subset \mathscr{K} .
$$

Set $\beta_{1}=\left\{\zeta^{*} \in \beta ; K\left(\cdot, \zeta^{*}\right) \in \operatorname{ex}\left[\mathscr{P}_{1}\right]\right.$. Then (14) means that

$$
\left\{K\left(\cdot, \zeta^{*}\right) ; \zeta^{*} \in \beta_{1}\right\}=\operatorname{ex}\left[\mathscr{P}_{1}\right] .
$$

By comparing topologies in $\beta \cup \Omega$ and $\mathscr{E}$, we deduce that by the mapping $T: \zeta^{*} \rightarrow K\left(\cdot, \zeta^{*}\right) \beta_{1}$ and ex $\left[\mathscr{P}_{1}\right]$ are homeomorphic. We set $d \mu\left(\zeta^{*}\right)=d \nu\left(T \zeta^{*}\right)$ for $\nu$ in (3). Then (3) can be rewritten as

$$
u=\int_{\beta_{1}} K\left(\cdot, \zeta^{*}\right) d \mu\left(\zeta^{*}\right)
$$


We have thus obtained the Martin representation theorem (cf. [9], Ito [6], Šur [18], etc.): There exists a bijective correspondence $u \leftrightarrow \mu$ related by (16) between $\mathscr{P}$ and the family $\{\mu\}$ of regular Borel measures $\mu$ on $\beta$ supported by $\beta_{1}$. We define the elliptic dimension of $\mathscr{P}$ at $\delta: z=0$, $\operatorname{dim} P$ in notation, by

$$
\operatorname{dim} P=\sharp\left(\operatorname{ex}\left[\mathscr{P}_{1}\right]\right)=\sharp \beta_{1}
$$

where \# denotes the cardinal number. If $\operatorname{dim} P=1$, then we say after Bouligand that the Picard principle is valid for $P$, or for the operator $L_{P}=\Delta-P$, at $\delta$. In this case we see by (16) that $\mathscr{K}$ contains a single element, i.e.

$$
\# \beta=1 \text {. }
$$

Conversely, since $\beta \supset \beta_{1} \neq \phi$, (18) implies that $\operatorname{dim} P=1$. Therefore the Picard principle is valid for $P$ at $\delta$ if and only if $\beta=\beta_{P}$ satisfies (18).

7. We denote by $\mathscr{P}(t)$ the space of nonnegative solutions of $L_{P} u=0$ on $\Omega_{t}: 0<|z|<t$ with vanishing boundary values on $\partial \Omega_{t}$. Thus $\mathscr{P}(1)=$ $\mathscr{P}$. We also denote by $\mathscr{B}(t)$ the Banach space of bounded solution of $L_{P} u=0$ on $\Omega_{t}$ with continuous boundary values on $\partial \Omega_{t}$ and hence $\mathscr{B}(1)$ $=\mathscr{B}$ (cf. no. 3). We shall show that $\operatorname{dim} P$ does not depend on $\Omega_{t}$ $(0<t \leq 1)$, i.e. there exists a positively linear bijective map between $\mathscr{P}(t)$ and $\mathscr{P}=\mathscr{P}(1)$ (cf. Ozawa [15]). Take an $s$ with $0<s<t<1$ and let $A$ be the operator from $C\left(\partial \Omega_{t}\right)$ onto $\mathscr{B}(t)$ such that $(A \varphi) \mid \partial \Omega_{t}=\varphi$. We also denote by $B \varphi$ for $\varphi \in C\left(\partial \Omega_{s}\right)$ the solution of $L_{P} u=0$ on $\Omega-\bar{\Omega}_{s}$ with boundary values $\varphi$ on $\partial \Omega_{s}$ and zero on $\partial \Omega$. We maintain that the mapping $\pi: \mathscr{P} \rightarrow \mathscr{P}(t)$ given by

$$
\pi u=u-A u
$$

is the required map. That $\pi u \geq 0$ for $u \in \mathscr{P}$ can be seen by the maximum principle and the way of constructing $A u$ as $\lim _{s \rightarrow 0} v_{s}$ where $v_{s}$ is the solution of $L_{P} u=0$ on $s<|z|<t$ with boundary values $u$ on $|z|=t$ and zero on $|z|=s$. Clearly $\pi$ is positively linear: $\pi(\lambda u)=\lambda \pi u$ for real numbers $\lambda \geq 0$ and $\pi\left(u_{1}+u_{2}\right)=\pi u_{1}+\pi u_{2}$. Suppose $\pi u_{1}=\pi u_{2}$. Then $u_{1}-u_{2}$ $=A\left(u_{1}-u_{2}\right)$ on $\Omega_{t}$ shows that $u_{1}-u_{2} \in \mathscr{B} \cap \mathscr{E}$, and by the maximum principle, $\sup _{\bar{\Omega}}\left|u_{1}-u_{2}\right|=\max _{\partial \Omega}\left|u_{1}-u_{2}\right|=0$, i.e. $\pi$ is injective. Let $v \in \mathscr{P}(t)$. Finding $u \in \mathscr{P}$ with 


$$
A(u-v)=u-v
$$

is nothing but the Sario principal function problem (see e.g. RodinSario [17]) and the solvability of (20) settles the surjectiveness of $\pi$. Consider

$$
T \varphi=A(B \varphi)
$$

for $\varphi \in C\left(\partial \Omega_{s}\right)$. By the maximum principle and the Harnack principle, we see that $T$ is a compact linear operator from $C\left(\partial \Omega_{s}\right)$ into itself. By the maximum principle for $A$ and $B$ (cf. no. 3), we see that $T \varphi=\varphi$ implies that $\varphi=0$. Therefore the Riesz-Schauder theory (cf. e.g. Yosida [20]) assures that there exists a unique $\varphi \in C\left(\partial \Omega_{s}\right)$ with $\varphi-T \varphi=v$. Then $B \varphi=A(B \varphi)+v$ on $\left(\partial \Omega_{t}\right) \cup\left(\partial \Omega_{s}\right)$ and therefore on $\Omega_{t} \cap\left(\Omega-\bar{\Omega}_{s}\right)$. Thus the function

$$
u(z)= \begin{cases}(B \varphi)(z) & \left(z \in \Omega-\bar{\Omega}_{s}\right) ; \\ (A(B \varphi))(z)+v(z) & \left(z \in \Omega_{t}\right)\end{cases}
$$

is well defined on $\Omega$, a solution of $L_{P} u=0$ on $\Omega$ with vanishing boundary values on $\partial \Omega$, and satisfies (20), i.e. $u-A u=v \geq 0$ on $\Omega_{t}$. The last means that $u$ is bounded from below by a constant, say $c$. Let $w_{s}$ be the solution of $L_{P} u=0$ on $0<s<|z|<1$ with boundary values 1 on $|z|=s$ and zero on $|z|=1$. Then $u-c w_{s} \geq 0$ for every small $s>0$. A fortiori $\lim _{s \rightarrow 0} w_{s}=0$ implies that $u \in \mathscr{P}$.

8. We associate with the operator $L_{P}=\Delta-P$ the operator $\hat{L}_{P}$ given by

$$
\hat{L}_{P} u(z)=\Delta u(z)+2 \nabla \log e_{P}(z) \cdot \nabla u(z)
$$

for $u \in C^{2}(\Omega)$ where $e=e_{P}$ is the $P$-unit. The importance of the $\hat{L}_{P}$ for the study of the Picard principle as well as the following duality is first observed by Heins [5] and later by Hayashi [4]. It is characteristic that the associated operator $\hat{L}$ does not contain the term of zero order differentiations even for the general, i.e. not necessarily selfadjoint, operator $L$ (cf. Nakai [14]). We say that the Riemann theorem is valid for $\hat{L}_{P}$ at $\delta: z=0$ if $\lim _{z \rightarrow 0} u(z)$ exists for every bounded solution $u$ of $\hat{L}_{P} u=0$ in a punctured neighborhood $0<|z|<t \leq 1$ of $\delta: z=0$. We maintain

THEOREM. The Picard principle is valid for the operator $L_{P}$ at $\delta$ if 
and only if the Riemann theorem is valid for the associated operator $\hat{L}_{P}$ at $\delta$.

Proof. We denote by $\hat{\mathscr{B}}$ the space of bounded solutions of $\hat{L}_{P} u=0$ on $\Omega$ with continuous boundary values on $\partial \Omega$. Observe that $u \rightarrow u / e$ is an isomorphism of $\mathscr{B}$ onto $\hat{\mathscr{B}}$ as Banach spaces, where $e=e_{P}$. Let $\Omega_{t}$ be $0<|z|<t(0<t<1)$ and $G_{t}(z, \zeta)$ be the Green's function of $L_{P} u=0$ on $\Omega-\bar{\Omega}_{t}$. Take an arbitrary $v \in \hat{\mathscr{B}}$ and let $u=e v \in \mathscr{B}$. Then

$$
u_{t}(z)=\frac{-1}{2 \pi} \int_{0}^{2 \pi}\left[\frac{\partial}{\partial r} G_{t}\left(r \mathrm{e}^{i \theta}, z\right) u\left(r \mathrm{e}^{i \theta}\right)\right]_{r=1} d \theta
$$

is a bounded solution of $L_{P} u=0$ on $\Omega-\bar{\Omega}_{t}$ with boundary values $u$ on $\partial \Omega$ and zero on $\partial \Omega_{t}$. Therefore $u=\lim _{t \rightarrow 0} u_{t}$ and $G=\lim _{t \rightarrow 0} G_{t}$ imply that

$$
u(z)=\frac{-1}{2 \pi} \int_{0}^{2 \pi}\left[\frac{\partial}{\partial r} G\left(r \mathrm{e}^{i \theta}, z\right) u\left(r \mathrm{e}^{i \theta}\right)\right]_{r=1} d \theta
$$

Dividing the both sides of the above by $e$, we obtain

$$
v(z)=-\int_{0}^{2 \pi}\left[\frac{\partial}{\partial r} K\left(r \mathrm{e}^{i \theta}, z\right) v\left(r \mathrm{e}^{i \theta}\right)\right]_{r=1} d \theta
$$

Since the right hand side of the above is continuous on $\beta \cup \Omega$, the same is true of $v$, i.e. $v$ can be extended uniquely to $\Omega^{*}$ so as to be a continuous function on $\Omega^{*}$, and in this sense we have

$$
\hat{\mathscr{B}} \subset C\left(\Omega^{*}\right) \text {. }
$$

In particular, $\hat{\mathscr{B}} \mid \beta \subset C(\beta)$. We next asserts that $\hat{\mathscr{B}} \mid \beta$ separates points in $\beta$, i.e. for any pair of distinct points $\zeta_{1}^{*}$ and $\zeta_{2}^{*}$ in $\beta$, there exists a $v \in \mathscr{B}$ such that $v\left(\zeta_{1}^{*}\right) \neq v\left(\zeta_{2}^{*}\right)$. If this were not the case, then (22) with $z$ replaced by $\zeta_{j}^{*}(j=1,2)$ would imply

$$
\int_{0}^{2 \pi}\left[\frac{\partial}{\partial r} k\left(r \mathrm{e}^{i \theta}\right)\right]_{r=1} v\left(e^{i \theta}\right) d \theta=0
$$

for every $v \in \hat{\mathscr{B}}$ and hence for every $v \in C(\partial \Omega)$ where $k(\zeta)=K\left(\zeta, \zeta_{1}^{*}\right)-$ $K\left(\zeta, \zeta_{2}^{*}\right)$, which vanishes continuously on $\partial \Omega$. Therefore $k \in C(\bar{\Omega})$ satisfies

$$
L_{P} k=0, \quad k\left(e^{i \theta}\right)=\left[\frac{\partial}{\partial r} k\left(r \mathrm{e}^{i \theta}\right)\right]_{r=1}=0 .
$$

By the uniqueness of the Cauchy problem we have $k \equiv 0$ on $\Omega$ (cf. e.g. 
Miranda [8]), which contradicts $\zeta_{1}^{*} \neq \zeta_{2}^{*}$. Since we have

$$
\min _{\zeta^{*} \in \beta} v\left(\zeta^{*}\right) \leq \lim _{z \rightarrow 0} \inf v(z) \leq \lim _{z \rightarrow 0} \sup v(z) \leq \max _{\zeta^{*} \in \beta} v\left(\zeta^{*}\right)
$$

for every $v \in \hat{\mathscr{B}}$, we conclude that $\lim _{z \rightarrow 0} v(z)$ exists for every $v \in \hat{\mathscr{B}}$ if and only if (18) is valid, which is equivalent to that $\operatorname{dim} P=1$. Since the above argument can be repeated if $\Omega$ is replaced by $\Omega_{t}: 0<|z|<t(0<t<1)$, the result in no. 7 assures that the theorem is valid.

9. By the general theory developed in nos. 1-8 the study of Picard principle for the operator $L_{P}$ is identical with the study of the Riemann theorem for the associated operator $\hat{L}_{P}$. We shall give a sufficient condition to assure the validity of the Riemann theorem for $\hat{L}_{P}$. Consider the condition

$$
\int_{\Omega}\left|\nabla \log e_{P}(z)\right|^{2} d x d y<\infty
$$

for the $P$-unit $e=e_{P}$ on $\Omega$. Denote by $\Omega_{t}$ the punctured disk $0<|z|<t$ $(0<t \leq 1)$. We start with

Proposition. If the condition (24) is satisfied, then any bounded solution $u$ of $\hat{L}_{P} u=0$ on $\bar{\Omega}_{t}(0<t<1)$ has a square integrable gradients on $\Omega_{t}$, i.e.

$$
\int_{\Omega_{t}}|\nabla u(z)|^{2} d x d y<\infty .
$$

Proof. Let $u_{s}$ be the bounded solution of $\hat{L}_{P} u=0$ on $\Omega_{t}-\bar{\Omega}_{s}(0<$ $s<t$ ) with boundary values $u$ on $\partial \Omega_{t}$ and zero on $\partial \Omega_{s}$. Then $v_{s}=e u_{s}$ is the bounded solution of $L_{P} u=0$ on $\Omega_{t}-\bar{\Omega}_{s}$ with boundary values $e u$ on $\partial \Omega_{t}$ and zero on $\partial \Omega_{s}$. Observe that $\left|v_{s}\right| \leq k e$ on $\Omega_{t}-\bar{\Omega}_{s}$ with $k=$ $\sup _{\bar{a}_{t}}|u|$. By no. $3, v=\lim _{s \rightarrow 0} v_{s}$ exists and is a solution of $L_{P} u=0$ on $\Omega_{t}$ with boundary values $e u$ on $\partial \Omega_{t}$. Moreover $|v| \leq k e$. On the other hand $e u$ is a bounded solution of $L_{P} u=0$ on $\Omega_{t}$ with boundary values $e u$ on $\partial \Omega_{t}$. Therefore $v=e u$, i.e. $u=\lim _{s \rightarrow 0} u_{s}$ uniformly on each compact subset of $\bar{\Omega}_{t}$. Since $u_{s}=u$ on $\partial \Omega_{t}$ which is of class $C^{1}$ on $\partial \Omega_{t}$, we deduce that $u_{s} \in C^{1}\left(\bar{\Omega}_{t}-\Omega_{s}\right)$ and

$$
\frac{\partial}{\partial t} u\left(t e^{i \theta}\right)=\lim _{s \rightarrow 0} \frac{\partial}{\partial t} u_{s}\left(t e^{i \theta}\right)
$$

uniformly on $[0,2 \pi]$. Therefore $\lim _{s \rightarrow 0} B\left(u_{s}\right)=B(u)$ where 


$$
B(u)=\int_{0}^{2 \pi} u\left(t e^{i \theta}\right) \frac{\partial}{\partial t} u\left(t e^{i \theta}\right) t d \theta
$$

We extend $u_{s}$ to $\Omega_{t}$ by setting $u_{s}=0$ on $\Omega_{s}$. Using the notation

$$
D(\varphi)=\int_{\Omega_{t}}|\nabla \varphi(z)|^{2} d x d y
$$

for sectionally $C^{1}$-functions $\varphi$ on $\Omega_{t}$ we deduce the identity

$$
B\left(u_{s}\right)=D\left(u_{s}\right)+\int_{\Omega_{t}-\bar{\Omega}_{s}} u_{s}(z) \Delta u_{s}(z) d x d y
$$

from the Stokes formula. Replacing $\Delta u_{s}$ by $-2 \nabla \log e \cdot \nabla u_{s}$ on $\Omega_{t}$ we have

$$
B\left(u_{s}\right)=D\left(u_{s}\right)-2 \int_{\Omega_{t}} u_{s}(z) \nabla \log e(z) \cdot \nabla u_{s}(z) d x d y .
$$

Observing that $\left|u_{s}\right| \leq k=\sup _{\bar{\Omega}_{t}}|u|$ on $\Omega_{t}$ we conclude that

$$
D\left(u_{s}\right) \leq\left|B\left(u_{s}\right)\right|+k \int_{\Omega_{t}}\left|2 \nabla \log e(z) \cdot \nabla u_{s}(z)\right| d x d y .
$$

On denoting by $m^{2}$ the four times of the quantity (24), the Schwarz inequality yields

$$
D\left(u_{s}\right) \leq\left|B\left(u_{s}\right)\right|+k m D\left(u_{s}\right)^{1 / 2}
$$

We now maintain that $\lim \sup _{s \rightarrow 0} D\left(u_{s}\right)<\infty$ and thus $\lim \inf _{s \rightarrow 0} D\left(u_{s}\right)<\infty$. If this were not the case there would exist a strictly decreasing zero sequence $\{s(n)\}$ in $(0,1)$ such that $\lim _{n \rightarrow \infty} D\left(u_{s(n)}\right)=\infty$. By (26) we have

$$
\left.D\left(u_{s(n)}\right)^{1 / 2} \leq \mid B u_{s(n)}\right) \mid \cdot D\left(u_{s(n)}\right)^{-1 / 2}+k m
$$

for $n=1,2, \cdots$. Since $\left|B\left(u_{s(n)}\right)\right| \rightarrow|B(u)|<\infty$, we arrive at a contradiction by letting $n \rightarrow \infty$ in the above inequality. By the Fatou lemma we finally conclude that

$$
\int_{\Omega_{t}}|\nabla u(z)|^{2} d x d y \leq \liminf _{s \rightarrow 0} \int_{\Omega_{t}}\left|\nabla u_{s}(z)\right|^{2} d x d y<\infty
$$

10. In view of the preceding proposition we shall next study the limit property of functions with (25). Consider a real valued continuous function $u$ on $\bar{\Omega}$. The behavior of $u$ in a small neighborhood of $\delta: z=0$ is regulated to a certain extent by the behavior of a pair of functions 


$$
\left\{\begin{array}{l}
M(r)=M(r ; u)=\max _{|z|=r} u(z) \\
m(r)=m(r ; u)=\min _{|z|=r} u(z)
\end{array}\right.
$$

for $r \in(0,1]$ as $r \rightarrow 0$. A necessary condition for the existence of $\lim _{z \rightarrow 0} u(z)$ is that the oscilation $M(r)-m(r)$ of $u$ on $|z|=r$ tends to zero as $r \rightarrow 0$. Concerning this we first prove the following

Proposition. If a function $u \in C^{1}(\Omega)$ has a square integrable gradient over $\Omega$, i.e. $\int_{\Omega}|\nabla u(z)|^{2} d x d y<\infty$, then there exists a closed subset $E$ of the interval $(0,1]$ with a finite logarithmic measure, i.e. $\int_{E} d \log r<\infty$, such that

$$
\lim _{r \in E, r \rightarrow 0}(M(r ; u)-m(r ; u))=0 .
$$

Proof. Fix an $r \in(0,1)$ and let $\theta_{M}$ and $\theta_{m}$ be in $(0,2 \pi]$ such that $u\left(r \mathrm{e}^{i \theta^{\theta t r}}\right)=M(r)$ and $u\left(r \mathrm{e}^{i \theta_{m}}\right)=m(r)$. Observe that $\lambda(r)=M(r)-m(r) \geq 0$ is continuous on $(0,1]$ and we have

$$
\lambda(r)=\int_{\theta_{m}}^{\theta_{M K}} \frac{\partial}{\partial \theta} u\left(r \mathrm{e}^{i \theta}\right) d \theta \leq \int_{0}^{2 \pi}\left|\frac{\partial}{\partial \theta} u\left(r \mathrm{e}^{i \theta}\right)\right| d \theta .
$$

By applying the Schwarz inequality to the right of the above we obtain

$$
\lambda(r)^{2} \leq 2 \pi \int_{0}^{2 \pi}\left|\frac{\partial}{\partial \theta} u\left(r \mathrm{e}^{i \theta}\right)\right|^{2} d \theta .
$$

Since $\left|\nabla u\left(r \mathrm{e}^{i \theta}\right)\right|^{2}=\left|\partial u\left(r \mathrm{e}^{i \theta}\right) / \partial r\right|^{2}+r^{-2}\left|\partial u\left(r \mathrm{e}^{i \theta}\right) / \partial \theta\right|^{2}$, we have

$$
\lambda(r)^{2} / r \leq 2 \pi \int_{0}^{2 \pi}\left|\nabla u\left(r \mathrm{e}^{i \theta}\right)\right|^{2} r d \theta .
$$

Integrating the both sides of the above on $(0,1)$ with respect to $d r$, we deduce

$$
\int_{0}^{1} \lambda(r)^{2} d \log r \leq 2 \pi \int_{\Omega}|\nabla u(z)|^{2} d x d y<\infty .
$$

We set $a_{n}=\int_{1 /(n+1)}^{1 / n} \lambda(r)^{2} d \log r \geq 0$ for $n=1,2, \ldots$ The inequality (29) yields $\sum_{1}^{\infty} a_{n}<\infty$. Choose a decreasing zero sequence $\left\{\varepsilon_{n}\right\}(n=1,2, \cdots)$ of positive numbers $\varepsilon_{n}$ such that 


$$
\sum_{n=1}^{\infty} \varepsilon_{n}^{-2} a_{n}<\infty
$$

Let $E_{n}=\left\{r \in[1 /(n+1), 1 / n] ; \lambda(r) \geq \varepsilon_{n}\right\}$ and $E=\bigcup_{1}^{\infty} E_{n}$. Then $E$ is a closed subset of $(0,1]$ and

$$
\begin{aligned}
\int_{E} d \log r & =\sum_{n=1}^{\infty} \varepsilon_{n}^{-2} \int_{E_{n}} \varepsilon_{n}^{2} d \log r \leq \sum_{n=1}^{\infty} \varepsilon_{n}^{-2} \int_{E_{n}} \lambda(r)^{2} d \log r \\
& \leq \sum_{n=1}^{\infty} \varepsilon_{n}^{-2} \int_{1 /(n+1)}^{1 / n} \lambda(r)^{2} d \log r=\sum_{n=1}^{\infty} \varepsilon_{n}^{-2} a_{n}<\infty,
\end{aligned}
$$

i.e. $E$ has a finite logarithmic measure. If $r \notin E$ and $r \in(0,1 / n]$, then $\lambda(r)<\varepsilon_{n}$, i.e. $\lim _{r \notin E, r \rightarrow 0} \lambda(r)=0$.

11. We state two direct consequences of the above proposition. Let $u \in C^{1}(\Omega)$ with finite square integrable gradient over $\Omega$. Suppose $u$ satisfies the maximum-minimum principle on each $\Omega_{r}: 0<|z|<r$ for every $r \in(0,1]$ in the following sense:

$$
\left\{\begin{array}{l}
M(r ; u)=\sup _{z \in \bar{a}_{r}} u(z) \\
m(r ; u)=\inf _{z \in \bar{a}_{r}} u(z)
\end{array}\right.
$$

for every $r \in(0,1]$. The conclusion in this case is that $\lim _{z \rightarrow 0} u(z)$ exists. To prove this first observe that, by $(30), M(r)$ is decreasing and $m(r)$ is increasing on $(0,1]$ as $r \rightarrow 0$. Therefore $\lambda(r)$ is decreasing on $(0,1]$ as $r \rightarrow 0$. A fortiori (28) implies that $\lim _{r \rightarrow 0} \lambda(r)=0$. Again by (30) we have

$$
0 \leq \limsup _{z \rightarrow 0} u(z)-\liminf _{z \rightarrow 0} u(z) \leq \lambda(r)
$$

for every $r \in(0,1]$ and therefore $\lim _{z \rightarrow 0} u(z)$ exists.

12. Again let $u \in C^{1}(\Omega)$ have a finite square integrable gradient over $\Omega$. Suppose $u$ has a 'mean limit' at $\delta: z=0$ in the following sense: there exists a point $z(r)$ on the circle $|z|=r$ for each $r \in(0,1]$ and a real number $\ell$ such that $\ell=\lim _{r \rightarrow 0} u(z(r))$. Then there exists a closed subset $E$ of $(0,1]$ with a finite logarithmic measure such that

$$
\lim _{|z| \notin E, z \rightarrow 0} u(z)=\ell .
$$

For the proof take the exceptional set $E$ in Proposition in no. 10. Observe that 


$$
|u(z)-u(z(|z|))| \leq \lambda(|z|)
$$

and the right hand side term of the above tends to zero as $z \rightarrow 0$ with $|z| \notin E$. Thus the desired conclusion can be deduced.

13. Combining Propositions in nos. 9 and 10 we obtain the following test for the Riemann theorem and hence for the Picard principle in terms of the $P$-unit $e=e_{P}$ :

THEOREM. The Riemann theorem is valid for $\hat{L}_{P}$ at $\delta$ and therefore the Picard principle is valid for the density $P$ on $\Omega$ at $\delta$ if the $P$-unit $e_{P}$ satisfies (24).

Proof. By Proposition in no. 9 any bounded solution $u$ of $\hat{L}_{P} u=0$ on $\bar{\Omega}_{t}(0<t<1)$ has square integrable gradient over $\Omega_{t}$. Moreover $u$ satisfies (30) for $r \in(0, t]$. In fact, let $v=e u$. Then $v$ is a bounded solution of $L_{P} u=0$ on $\bar{\Omega}_{t}$ and

$$
m(r ; u) e \leq v \leq M(r ; u) e
$$

on $\partial \Omega_{r}$ for every $r \in(0, t]$. Therefore the same inequality is valid on $\bar{\Omega}_{r}$ (cf. no. 3) and we conclude that $m(r) \leq u \leq M(r)$ on $\bar{\Omega}_{r}$, which implies (30). A fortiori, the result in no. 11 assures the existence of $\lim _{z \rightarrow 0} u(z)$, i.e. the Riemann theorem is valid for $\hat{L}_{P}$ at $\delta$. By Theorem in no. 8 we also conclude the validity of the Picard principle for $P$ at $\delta$.

14. Although the following is essentially identical with the criterion in Nakai [11] and will not be made use of in the main context in this paper, we include it here for comparison:

THEOREM. The Riemann theorem is valid for $\hat{L}_{P}$ at $\delta$ and therefore the Picard principle is valid for the density $P$ at $\delta$ if the $P$-unit $e_{P}$ satisfies

$$
\limsup _{z \rightarrow 0} e_{P}(z)>0 .
$$

Proof. Let $u$ be any bounded solution of $\hat{L}_{P} u=0$ on $\bar{\Omega}_{t}(0<t<1)$ and $v=e u$. By the fact that $v=\lim _{s \rightarrow 0} v_{s}$ as in the proof of Proposition in no. 9, we have $V(v) \leq \lim _{\inf _{s \rightarrow 0}} V\left(v_{s}\right)$ where

$$
V(\varphi)=\int_{\Omega_{t}}\left[|\nabla \varphi(z)|^{2}+P(z) \varphi(z)^{2}\right] d x d y
$$


for sectionally $C^{1}$ functions $\varphi$ on $\Omega_{t}$ and we have set $v_{s}=0$ on $\Omega_{s}$ $(0<s<t)$. Since $V$ is the variation whose Euler-Lagrange equation is $\Delta u=P u$, we see that $V\left(v_{s}\right) \leq V\left(v_{s_{0}}\right)<\infty$ for every $s \in\left(0, s_{0}\right]\left(0<s_{0}<t\right)$. Therefore, in particular, $v$ has a square integrable gradient on $\Omega_{t}$ and the same is true of $e$. For a function $\varphi \in C\left(\bar{\Omega}_{t}\right)$ we associate a function

$$
\varphi^{*}(r)=\frac{1}{2 \pi} \int_{0}^{2 \pi} \varphi\left(r \mathrm{e}^{i \theta}\right) d \theta
$$

on $(0, t]$. If $\varphi$ is bounded, subharmonic, and nonnegative, then $\lim _{r \rightarrow 0} \varphi^{*}(r)$ exists and identical with $\lim \sup _{z \rightarrow 0} \varphi(z)$ (cf. e.g. Tsuji [19]). This is the case for $\varphi=e$ and also for $v+k e$ for sufficiently large $k>0$. Since $(v+k e)^{*}=v^{*}+k e^{*}$, we conclude that $\ell_{e}=\lim _{r \rightarrow 0} e^{*}(r)>0$ and $\ell_{v}=$ $\lim _{r \rightarrow 0} v^{*}(r)$ exist. For each $r \in(0, t]$ there exists a $z(r)$ in $|z|=r$ with $e(z(r))=e^{*}(r)$ and the same is true of $v$. We can apply the result in no. 12 to $e$ and $v$. Let $E_{e}$ and $E_{v}$ be the exceptional sets as in no. 12 for $e$ and $v$. Then $E=E_{e} \cup E_{v}$ can also be used for the exceptional set both for $e$ and $v$, and $\int_{E} d \log r<\infty$. Since $u=v / e$, we have

$$
\lim _{|z| \notin E, z \rightarrow 0} u(z)=\ell_{v} / \ell_{e}
$$

As in no. 13, (30) is valid for $u$. This with (32) implies that $\lim _{z \rightarrow 0} u(z)$ $=\ell_{v} / \ell_{e}$, i.e. the Riemann theorem is valid for $\hat{L}_{P}$ at $\delta$.

15. We are ready to prove the main theorem stated in the introduction. The key step in our proof is the following evaluation of the square integral of the gradient of the logarithm of the $P$-unit $e=e_{P}$ :

Proposition. The $P$-unit $e_{P}$ of any density $P$ on $\Omega$ satisfies the following inequality

$$
\int_{\Omega}\left|\nabla \log e_{P}(z)\right|^{2} d x d y \leq \int_{\Omega} P(z)\left(1-e_{P}(z)\right) d x d y .
$$

Proof. Let $e_{t}$ be the $P$-unit of $L_{P} u=0$ on $\Delta_{t}: t<|z|<1(0<t<1)$, i.e. $e_{t}$ be the solution of $L_{P} u=0$ on $\Delta_{t}$ with boundary values 1 on $\partial \Delta_{t}$. Since $\left\{e_{t}\right\}$ is decreasing as $t \rightarrow 0$, it converges to a bounded solution of $L_{P} u=0$ on $\Omega$ with boundary values 1 on $\partial \Omega$, and a fortiori, by no. 3 , $e=\lim _{t \rightarrow 0} e_{t}$ uniformly on each compact subset of $\bar{\Omega}$, and similarly $|\nabla e|=$ $\lim _{t \rightarrow 0}\left|\nabla e_{t}\right|$ uniformly on each compact subset of $\bar{\Omega}$. By the Stokes formula we have 


$$
\int_{0}^{2 \pi}\left[\frac{1}{e_{t}\left(r \mathrm{e}^{i \theta}\right)} r \frac{\partial}{\partial r} e_{t}\left(r \mathrm{e}^{i \theta}\right)\right]_{t}^{1} d \theta=\int_{\Delta_{t}} \nabla \frac{1}{e_{t}(z)} \cdot \nabla e_{t}(z) d x d y+\int_{\Delta_{t}} \frac{1}{e_{t}(z)} \Delta e_{t}(z) d x d y .
$$

Once more by the Stokes formula the left hand side of the above equals

$$
\int_{0}^{2 \pi}\left[r \frac{\partial}{\partial r} e_{t}\left(r \mathrm{e}^{i \theta}\right)\right]_{t}^{1} d \theta=\int_{\Delta_{t}} \Delta e_{t}(z) d x d y=\int_{\Delta_{t}} P(z) e_{t}(z) d x d y
$$

Therefore we obtain the identity

$$
\int_{\Delta_{t}} P(z) e_{t}(z) d x d y=-\int_{\Delta_{t}}\left|\nabla \log e_{t}(z)\right|^{2} d x d y+\int_{\Delta_{t}} P(z) d x d y
$$

On extending $e_{t}$ to $\Omega$ by setting $e_{t}=1$ on $\Omega-\Delta_{t}$ we conclude that

$$
\int_{\Omega}\left|\nabla \log e_{t}(z)\right|^{2} d x d y=\int_{\Omega} P(z)\left(1-e_{t}(z)\right) d x d y
$$

Observe that the integrand of the right hand side of the above is increasing as $t \rightarrow 0$. Therefore the Lebesgue theorem yields

$$
\lim _{t \rightarrow 0} \int_{\Omega} P(z)\left(1-e_{t}(z)\right) d x d y=\int_{\Omega} P(z)(1-e(z)) d x d y
$$

On taking the inferior limit as $t \rightarrow 0$ on the both sides of (34) and using the Fatou lemma, we deduce that

$$
\begin{aligned}
\int_{\Omega} P(z)(1-e(z)) d x d y & =\liminf _{t \rightarrow 0} \int_{\Omega}\left|\nabla \log e_{t}(z)\right|^{2} d x d y \\
& \geq \int_{\Omega}|\nabla \log e(z)|^{2} d x d y
\end{aligned}
$$

16. Proof of the main theorem. Suppose $P$ is a finite density on $\Omega$, i.e. (1) is satisfied. By (33), the condition (1) implies (24). By the theorem in no. 13, we conclude that the Picard principle is valid for $P$. The proof of the main theorem is herewith complete.

17. The condition (24) in Theorem in no. 13 is, at least superficially, better than the condition (1) in the main theorem. Between these two conditions there are involved two gaps: $\int_{\Omega}|\nabla \log e(z)|^{2} d x d y \leq$ $\int_{\Omega} P(z)(1-e(z)) d x d y$ and $\int_{\Omega} P(z)(1-e(z)) d x d y \leq \int_{\Omega} P(z) d x d y$. At this moment we cannot discuss whether the former of the above two inequalities are in fact strict in the essential sense, while both sides of the 
latter are simultaneously finite or infinite. The reason why we did not enlist the theorem in no. 13 as the main theorem, in spite of the fact that it is better result than the one stated in the introduction, is that the condition (1) is more explicit and seems to be tractable in practical applications than (24).

The condition (31) in Theorem in no. 14 is also implicit. However we can see that it is equivalent to

$$
\int_{\Omega-X} P(z) \log \frac{1}{|z|} d x d y<\infty
$$

for some closed subset $X=X_{P}$ in $\Omega$ which is thin at $z=0$, i.e. the point $z=0$ is an irregular boundary point for the harmonic Dirichlet problem for the open set $\Omega-X$. In fact, it was shown in [11] that (35) implies (31). Conversely suppose that (31) is valid, i.e. $\ell=\lim \sup _{z \rightarrow 0} e(z)>0$. Then for any $\varepsilon \in(0, \ell)$ the set $X=\{z \in \Omega ; e(z) \leq \varepsilon\}$ is closed in $\Omega$ and thin at $z=0$ (cf. e.g. Brelot [2]). Observe that $e(z)$ satisfies the integral equation

$$
e(\zeta)+\frac{1}{2 \pi} \int_{\Omega} e(z) P(z) \log \left|\frac{1-\bar{\zeta} z}{z-\zeta}\right| d x d y=1
$$

for every $\zeta \in \Omega$. Therefore

$$
\frac{1}{2 \pi} \int_{\Omega-x} P(z) \log \left|\frac{1-\bar{\zeta} z}{z-\zeta}\right| \leq 1 / \varepsilon
$$

for any $\zeta \in \Omega$. On letting $\zeta \rightarrow 0$ with using the Fatou lemma in the above we deduce (35). Thus the theorem in no. 14 is equivalent to the main theorem in [11]:

Theorem. The Picard principle is valid for a density $P$ at $\delta$ if $P$ satisfies the condition (35).

Clearly the conditions (1) and (35) are independent and thus both of them are not necessary for the validity of the Picard principle. Actually both of them cannot take care of the simple density $P(z)=|z|^{-2}$ for which the validity of Picard principle is known (cf. Nakai [10]). Except for rotation free densities $P$, i.e. densities $P$ with $P(z) \equiv P(|z|)$ on $\Omega$, the problem of determining $P$ with $\operatorname{dim} P=1$ seems to be very far from being complete. For the Picard principle for rotation free densities, 
refer to e.g. Brelot [1], Nakai [10,12,13], Kawamura-Nakai [7], etc.

\section{REFERENCES}

[1] M. Brelot: Étude des l'équation de la chaleur $\Delta u=c(M) u(M), c(M) \geqq 0$, au voisinage d'un point singulier du coefficient, Ann Éc. Norm. Sup., 48 (1931), 153-246.

[ 2 ] — : Lectures on Potential Theory, Tata Inst. Fund. Res., 1960.

[ 3 ] N. Dunford and L. Schwartz: Linear Operators, I, Interscience Publishers, 1957.

[4] K. Hayashi: Les solutions positives de l'equation $\Delta u=P u$ sur une surface de Riemann, Kōdai Math. Sem. Rep., 13 (1961), 20-24.

[5] M. Heins: Riemann surfaces of infinite genus, Ann. Math., 55 (1952), 296-317.

[6] S. Itô: Martin boundary for linear elliptic differential operators of second order in a manifold, J. Math. Soc. Japan, 16 (1964), 307-334.

[ 7 ] M. Kawamura and M. Nakai: A test of Picard principle for rotation free densities, II, J. Math. Soc. Japan, 28 (1976), 323-342.

[8] C. Miranda: Partial Differential Equations of Elliptic Type, Springer, 1970.

[ 9 ] M. Nakai: The space of nonnegative solutions of the equation $\Delta u=P u$ on a Riemann surface, Kōdai Math. Sem. Rep., 12 (1960), 151-178.

[10] — : Martin boundary over an isolated singularity of rotation free density, J. Math. Soc. Japan, 26 (1974), 483-507.

[11] — : A test for Picard principle, Nagoya Math. J., 56 (1974), 105-119.

[12] - : A remark on Picard principle, Proc. Japan Acad., 50 (1974), 806-808.

[13] _ - A test of Picard principle for rotation free densities, J. Math. Soc. Japan, 27 (1975), 412-431.

[14] —_: Picard principle and Riemann Theorem, Tôhoku Math. J., 28 (1976), 277292.

[15] M. Ozawa: Some classes of positive solutions of $\Delta u=P u$ on Riemann surfaces, I/II, Kōdai Math. Sem. Rep., 6/7 (1954)/(1955), 121-126/15-20.

[16] R. Phelps: Lectures on Choquet's Theorem, Van Nostrand Math. Studies \#7, 1965.

[17] B. Rodin and L. Sario: Principal Functions, Van Nostrand, 1968.

[18] M. Šur: The Martin boundary for a linear elliptic second order operator, Izv. Akad. Nauk SSSR, 27 (1963), 45-60 (Russian).

[19] M. Tsuji: Potential Theory in Modern Function Theory, Maruzen, 1959.

[20] K. Yosida: Functional Analysis, Springer, 1965.

Department of Mathematics

Nagoya Institute of Technology

Gokiso, Showa, Nagoya 466

Japan 